ACS Macro Lett. 2016 February 16; 5(2): 229-233. doi:10.1021/acsmacrolett.5b00923.

\title{
Visible-Light-Initiated Thiol-Michael Addition Polymerizations with Coumarin-Based Photobase Generators: Another Photoclick Reaction Strategy
}

\author{
Xinpeng Zhang ${ }^{\S, \dagger}$, Weixian $\mathrm{Xi}^{\S}, \dagger$, Chen Wang ${ }^{\dagger}$, Maciej Podgórski ${ }^{\dagger, \ddagger}$, and Christopher N. \\ Bowman ${ }^{*} \dagger$ \\ †Department of Chemical and Biological Engineering, University of Colorado, UCB 596, Boulder, \\ Colorado 80309, United States łFaculty of Chemistry, Department of Polymer Chemistry, MCS \\ University, pl. Marii Curie-Skłodowskiej 5, 20-031 Lublin, Poland
}

\begin{abstract}
An efficient visible-light-sensitive photobase generator for thiol-Michael addition reactions was synthesized and evaluated. This highly reactive catalyst was designed by protecting a strong base (tetramethyl guanidine, TMG) with a visible-light-responsive group which was a coumarin derivative. The coumarin-coupled TMG was shown to exhibit extraordinary catalytic activity toward initiation of the thiol-Michael reaction, including thiol-Michael addition-based polymerization, upon visible-light irradiation, leading to a stoichiometric reaction of both thiol and vinyl functional groups. Owing to its features, this visible-light photobase generator enables homogeneous network formation in thiol-Michael polymerizations and also has the potential to be exploited in other visible-light-induced, base-catalyzed thiol-click processes such as thiolisocynate and thiol-epoxy network-forming reactions.
\end{abstract}

\section{Graphical abstract}

\footnotetext{
*Corresponding Author: christopher.bowman@colorado.edu.

$\S_{\text {Author Contributions: }}$

Xinpeng Zhang and Weixian Xi contributed equally to this work

Supporting Information

The Supporting Information is available free of charge on the ACS Publications website at DOI: 10.1021/acsmacrolett.5b00923.

Experimental section including materials, coumarin-TMG and coumarin-hexylamine synthesis, and characterization techniques and Figures S1-S9 (PDF)

Notes

The authors declare no competing financial interest.
} 


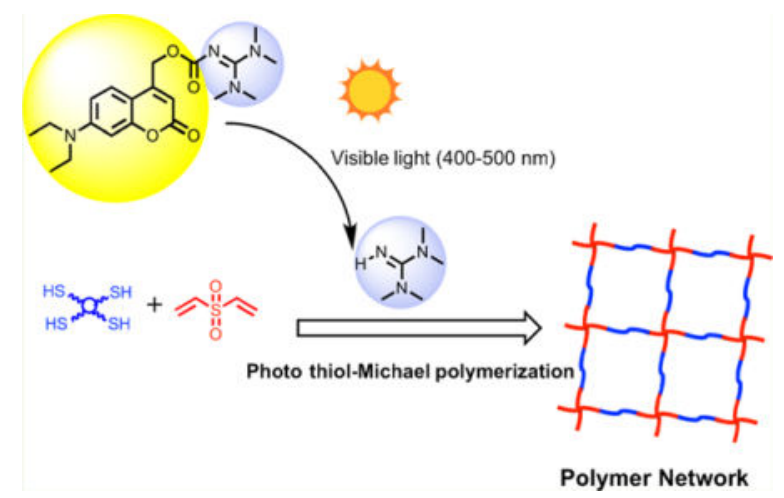

Since the introduction of "Click" chemistry in 2001, this concept has greatly affected the development of functional materials leading to strong interdisciplinary connections between the fields of chemistry, biology, and materials science. ${ }^{1}$ Among the family of click chemistry reactions, the thiol-Michael addition "click" reaction is a particularly useful tool in materials science, which allows for facile fabrication of cross-linked polymeric materials with high yields, rapid reaction rates, and the ability to be conducted at benign reaction conditions. ${ }^{2-5}$ Owing to these advantages, the thiol-Michael coupling has been widely implemented in different applications, such as surface functionalization, ${ }^{6}$ dendrimer synthesis, ${ }^{2,7}$ microparticle synthesis, ${ }^{8}$ polymer network synthesis, ${ }^{7,9-11}$ and biochemical modifications. ${ }^{4}$ However, compared with other "click" coupling reactions, such as the radical-mediated thiol-ene or thiol-yne reaction ${ }^{2-4}$ and the $\mathrm{Cu}(\mathrm{I})$-catalyzed alkyne-azide reaction, ${ }^{12,13}$ it is still challenging to achieve an ideal photoinitiating system that would provide spatial and temporal control over the thiol-Michael reaction, which inherently precludes a wide range of materials science applications.

Both bases (e.g., hexylamine, triethylamine) or nucleophiles (e.g., dimethyl phenyl phosphine, triphenyl phosphine) catalyze the thiol-Michael reaction efficiently through different initiating mechanisms. ${ }^{14}$ To achieve photoinduced control over thiol-Michael reactions, photorelease of bases or nucleophiles to trigger the process has been implemented as the most straightforward method. ${ }^{15-19}$ To achieve efficient light-induced anion-mediated reactions, different photocatalyzing systems, most often photobase generators (PBGs), have been designed and studied in the past few decades. ${ }^{20-23}$ These PBG catalyzing systems have been applied not only in base-catalyzed thiol-Michael processes but also in other anionmediated reactions such as ring-opening polymerization, ${ }^{22}$ polyamide fabrication, polythiourethane formation, etc.

Typical PBGs release primary or secondary amines upon irradiation which are relatively weak catalysts for the thiol-Michael reaction. ${ }^{15}$ Therefore, the "super-base" guanidine and its derivatives, such as 1,5,7-triazabicyclo[4.4.0]dec-5-ene (TBD) or tetramethylguanidine (TMG), have recently attracted researchers' attention because of their high basicity (TMG $\left.\mathrm{p} K_{\mathrm{a}}=13.6\right)$ which results in improved catalytic efficiency in anion-mediated polymerizations. ${ }^{22,24}$ To enable the base protection (or basicity reduction), varied photosensitive chemical groups, such as nitrophenyl propyl, ${ }^{25}$ phenylglyoxylic acid, ${ }^{15}$ and tetraphenylborate, ${ }^{22}$ have been utilized. However, for current PBGs, there remain several 
inherent problems that need to be overcome through photobase molecular design and synthesis. One major challenge with most PBGs is that they have no absorption above 400 $\mathrm{nm}$, and the base generation can only be triggered by UV light. Compared to visible-light initiators, UV responsive catalysts require high photon energy, often achieve reduced curing depths, and may also cause irreversible damage to UV-sensitive cells or tissues in biochemical applications. Various approaches have been implemented to achieve visiblelight-sensitive PBGs. For instance, combinations of some UV-responsive PBGs (usually ionic complexes of protonated bases and photosensitive anions) and visible-light-active sensitizers (e.g., thioxanthone (TX), coumarin derivatives) were utilized to achieve photoinitiating cocatalyst systems that possess redshifted absorption $(>400 \mathrm{~nm}) .{ }^{26}$ Using energy transferred from triplet states of the excited sensitizers, these UV-sensitive PBGs undergo photocleavage under visible-light irradiation. ${ }^{27}$ However, other problems may arise in these systems due to the generation of radical species from these sensitizers. Especially in thiol-Michael reactions, radical generation by the sensitizers will promote radical-mediated homopolymerization, which would reduce the ultimate conversions and thus affect the system's orthogonality and "click" nature. ${ }^{28}$ Also, the use of ionic complexes is limited to hydrophilic monomer mixtures only, as these photosensitive salts exhibit poor solubility in nonpolar or low polarity comonomer systems.

Herein, we report the synthesis and implementation of coumarin-TMG as an enhanced PBG including (but not predominating) a radical path of reaction for visible-light-induced thiolMichael addition. Coumarin and its derivatives have been commonly used as visible-light sensitizers and protecting groups for alcohols or amines and were widely exploited in biomaterial and surface patterning. ${ }^{23,29}$ The wide range of applications of coumarin derivatives are attributed to their outstanding properties such as visible-light sensitivity, biological compatibility, and insignificant radical-based side reactions. ${ }^{30,31}$ The photocaged superbase here is referred to as coumarin-protected tetramethylguanidine (TMG), a strong Lewis base, which efficiently initiates the thiol-Michael reaction at extremely low levels of the free TMG. The synthetic route used to create the coumarin-TMG is presented in Scheme 1a. All four steps are conducted under mild conditions with a reasonable $20-30 \%$ overall yield.

The mechanism for photorelease of TMG is described in Scheme $1 \mathrm{~b} .{ }^{32}$ Upon visible-light irradiation, excitation takes place to the lowest excited singlet state. After that, the exited PBG can either undergo unproductive, nonradiative decay or fluorescence or it can undergo the desired, productive heterolysis process. Heterolytic $\mathrm{C}-\mathrm{O}$ bond cleavage leads to the formation of the key intermediate; i.e., it creates a tightly held ion pair (coumarinylmethyl cation and the corresponding leaving group). By reacting directly with nucleophiles or even solvent, the cation transforms into a new, stable coumarylmethyl product. In the case of the released carbamate anion, essential decarboxylation occurs and leads to the formation of carbon dioxide and tetramethyl guanidine (TMG).

By applying time-dependent UV-vis measurements, the photoinitiated behavior of coumarin-TMG was analyzed to confirm the photodecarboxylation process upon irradiation (Figure 1a). By utilizing $50 \mathrm{~mW} / \mathrm{cm}^{2}, 400-500 \mathrm{~nm}$ light irradiation, the absorption spectra of coumarin-TMG in methanol changed due to the phototriggered transformation of the 
coumarin structure. The lamp emission spectra and UV absorption of coumarin-TMG are compared in Figure S1. With increasing irradiation time, a decrease of the peak at $375 \mathrm{~nm}$ and increase of the peak at $268-340 \mathrm{~nm}$ were observed. This photobleaching transformation is due to the photolysis process, which leads to the generation of TMG, carbon dioxide, and coumarin residues. A slightly different bleaching photobehavior was observed in an acetonitrile solution of coumarin-TMG which indicates solution influence on the photolysis process (Figure S2). To understand better the effects of solvent on the photoinduced coumarin reaction behavior, 1H NMR was utilized, and photolysis of coumarin-TMG was monitored in different deuterated solvents $\left(\mathrm{CDCl}_{3}, \mathrm{CD}_{3} \mathrm{CN}\right.$, and $\left.\mathrm{CD}_{3} \mathrm{OD}\right)$ (Figure $\mathrm{S} 3$ ). The cleavage of coumarin-TMG was confirmed by monitoring the decreasing peak around 2.9 ppm, which corresponds to the methyl group in the protected tetramethylguanidine. ${ }^{1} \mathrm{H}$ NMR results for different solvents indicate that the proticity of solvents greatly affects the photodecomposition rate. Polar protic solvents (e.g., $\mathrm{CH}_{3} \mathrm{OH}, \mathrm{H}_{2} \mathrm{O}$, etc.) enable the fastest photodecomposition. In contrast, photocleavage is much slower in nonprotic conditions (e.g., $\mathrm{CH}_{3} \mathrm{CN}, \mathrm{CH}_{2} \mathrm{Cl}_{2}, \mathrm{CHCl}_{3}$, etc.), and polar environments do not significantly promote the reaction.

The photorelease of $\mathrm{CO}_{2}$ can be confirmed by FT-IR spectra as a formation and continuous increase in intensity of a new peak at $2200 \mathrm{~cm}^{-1}$ during the photoinitiated thiol-Michael polymerization. Furthermore, FT-IR was also applied to study the photodecarboxylation process by monitoring carbamate bond decomposition. Upon visible-light irradiation, the coumarin-TMG was consumed quantitatively in about $7 \mathrm{~min}$, which indicated a fast photolysis rate of coumarin-TMG in highly concentrated conditions (Figure S3). The quantum yield calculated by this method was $0.044( \pm 0.004)$ (Figure S4), which showed similar value to the previous reports on coumarin protecting groups. ${ }^{33}$ For the application in biochemistry and materials, this low quantum yield can be compensated by its longer wavelength absorption, extending to the $400 \mathrm{~nm}$ region and high absorption coefficient.

To detect the generation of free TMG during visible-light irradiation, a colorimetric method, which utilizes phenol red as a base indicator, was applied (Figure 1b). Coumarin-TMG ( $5 \times$ $10^{-3} \mathrm{M}$ ) in methanol was used. For each experiment, different visible-light irradiation time scales have been applied, and $10^{-3} \mathrm{M}$ phenol red was added afterward. The absorption spectra for each sample were collected. With increasing irradiation time of visible light $(400-500 \mathrm{~nm})$, a new absorption band arose around $567 \mathrm{~nm}$, which corresponds to the deprotonation of phenol red by the photoreleased base. This result clearly indicated that the coumarin-TMG underwent a photolysis process and released a free Lewis base (TMG) upon visible-light irradiation.

After the photolysis and light-triggered base formation of coumarin-TMG had been demonstrated, different monothiols were combined with divinyl sulfone as model substrates to study the catalyzing efficiency of the photobase at hand in the thiol-Michael reactions (Table 1). The thiol-vinyl sulfone reaction was monitored in real time FT-IR. During these experiments, the decreasing thiol peak around $2650 \mathrm{~cm}^{-1}$ was chosen as an indicative measure of the progress of the reaction. ${ }^{1} \mathrm{H}$ NMR was used to confirm the ultimate yields of the photoinduced thiol-Michael coupling reactions (Figure S5). The results in Table 1 indicate that with $2 \mathrm{wt} \%$ catalyst loading in the analyzed systems near-quantative 
conversions were achieved in a short period of time (less than $20 \mathrm{~min}$ ). In addition, entries 6 and 7 indicate that the photolabile catalyst and visible-light induction are both necessary to trigger the reaction.

Because of the initial base presence after irradiation, it is expected that the anion-mediated thiol-Michael reaction will continue in the dark even after cessation of irradiation. Moreover, this reaction is negligibly influenced by oxygen or quenched by radical-based side reactions. This living character of the thiol-Michael reaction would open up the possibility to achieve relatively high substrate conversions with limited light exposure times. To demonstrate this concept, butyl thiolglycolate and DVS were used as the thiol-Michael reactants, and a dark cure test was performed as depicted in Figure 2a. Compared to the continuous irradiation, the 2 min exposure experiment exhibits a distinctive and continuing, although relatively slower, reaction rate in the dark. However, it has clearly been shown that the thiol-Michael reaction continues from $50 \%$ (when the light was turned off) to over $90 \%$ conversion in 30 min. Though this test was conducted on a model non-cross-linking system, it reveals that the coumarin-TMG has the potential to achieve dark cure in the network-forming polymerizations.

As a materials synthesis example, coumarin-TMG was implemented as the photocatalyst for thiol-Michael polymerization of PETMP and DVS (Figure 2b). Varied amounts of coumarinTMG were applied in the stoichiometric PETMP/DVS formulations. As expected, in the same irradiation conditions $\left(50 \mathrm{~mW} / \mathrm{cm}^{2}, 400-500 \mathrm{~nm}\right)$, the system with a higher photobase loading exhibited a faster reaction rate. The ultimate thiol conversion was found to be around $80-90 \%$ due to vitrification of the polymer. Additionally, coumarin-protected hexylamine was also synthesized and compared with the coumarin-TMG. The UV absorption spectrum and extinction coefficient of the coumarin-hexylamine are similar to coumarin-TMG (Figure S6). However, with the same catalyst amount in the system, the coumarin-TMG catalyzed the thiol-Michael addition polymerization at a significantly faster reaction rate as compared to that of the coumarin-hexylamine. The differences in catalytic activity between coumarin-TMG and coumarin-hexylamine arise from the significant difference in the basicity of the released bases, i.e., TMG and hexylamine.

Finally, the mechanical properties of visible-light-triggered thiol-Michael polymers were evaluated. The $0.5 \mathrm{~mm}$ thick film made of a stoichiometric mixture of PETMP and DVS and cured in the presence of $2 \mathrm{wt} \%$ of coumarin-TMG was tested in DMA experiments (Figure 3a). The FT-IR test on the corresponding coumarin-TMG catalyzed the thiol-Michael polymerization and demonstrated almost the same thiol and vinyl group conversion which proves the formation of an orthogonally cross-linked network. From the DMA data, the relatively narrow $\tan \delta$ peaks indicate that a homogeneous polymer network was obtained in this photopolymerization process. Furthermore, being an effective PBG, the coumarin-TMG is successfully employed to phototrigger thio-isocynate or thiol-epoxide reactions including polymerizations. As an example, the coumarin-TMG was attempted in the anion-mediated thiol-epoxy polymerization, which is also widely exploited in various material science applications. PETMP and bisphenol A diglycidyl ether (diepoxy) were chosen as substrates. The polymer network formation and rapid polymerization were also observed in the FT-IR, again by monitoring the thiol functional group decay (Figure S7). As further depicted in 
Figure $3 \mathrm{~b}$, a vary narrow $\tan \delta$ peak was also obtained for the coumarin-TMG-catalyzed thiol-epoxy resin.

In conclusion, an efficient visible-light-sensitive photobase generator, i.e., coumarin-TMG, has been successfully synthesized, and its photobase-generating behavior as well as photolysis were studied in detail. Furthermore, the model thiol-Michael reactions of different monofunctional thiols and DVS were conducted to evaluate the catalytic activity of the photobase. It was also demonstrated that coumarin-TMG efficiently catalyzes other anion-mediated reactions under relatively mild conditions, such as thiol-epoxy and thiolisocyanate reactions. The photobase reported in this work enables spatiotemporal control over the initiation with visible light and allows for dark cure at short exposure times. As a whole, its use is expected to expand the photobase toolbox for base-catalyzed reactions in polymer science, biochemistry, and surface coating applications that demand mild reaction conditions under harmless visible-light irradiation.

\section{Supplementary Material}

Refer to Web version on PubMed Central for supplementary material.

\section{Acknowledgments}

The work was supported by National Institutes of Health (Grant 1U01DE023777-01) and the Industry/University Cooperative Research Center for Fundamentals and Applications of Photopolymerizations.

\section{References}

1. Xi WX, Scott TF, Kloxin CJ, Bowman CN. Adv Funct Mater. 2014; 24:2572.

2. Hoyle CE, Lowe AB, Bowman CN. Chem Soc Rev. 2010; 39:1355. [PubMed: 20309491]

3. Kolb HC, Finn MG, Sharpless KB. Angew Chem, Int Ed. 2001; 40:2004.

4. Nair DP, Podgorski M, Chatani S, Gong T, Xi WX, Fenoli CR, Bowman CN. Chem Mater. 2014; 26:724.

5. Xi WX, Wang C, Kloxin CJ, Bowman CN. ACS Macro Lett. 2012; 1:811.

6. Rizzi SC, Ehrbar M, Halstenberg S, Raeber GP, Schmoekel HG, Hagenmuller H, Muller R, Weber FE, Hubbell JA. Biomacromolecules. 2006; 7:3019. [PubMed: 17096527]

7. Chatani S, Podgorski M, Wang C, Bowman CN. Macromolecules. 2014; 47:4894.

8. Wang C, Podgorski M, Bowman CN. Mater Horiz. 2014; 1:535.

9. Podgorski M, Chatani S, Bowman CN. Macromol Rapid Commun. 2014; 35:1497. [PubMed: 24965270]

10. Chatani S, Nair DP, Bowman CN. Polym Chem. 2013; 4:1048.

11. Liu JC, Rad IY, Sun F, Stansbury JW. Polym Chem. 2014; 5:227.

12. Gong T, Adzima BJ, Baker NH, Bowman CN. Adv Mater. 2013; 25:2024. [PubMed: 23401189]

13. Ritter SC, Konig B. Chem Commun. 2006; 4694

14. Chan JW, Hoyle CE, Lowe AB, Bowman M. Macromolecules. 2010; 43:6381.

15. Salmi H, Allonas X, Ley C, Defoin A, Ak A. Polym Chem. 2014; 5:6577.

16. Jensen KH, Hanson JE. Chem Mater. 2002; 14:918.

17. Chae KH, Jang DJ. Macromol Symp. 1999; 142:173.

18. Sarker AM, Kaneko Y, Neckers DC. Chem Mater. 2001; 13:3949.

19. Chae KH, Jang HJ. J Polym Sci, Part A: Polym Chem. 2002; 40:1200. 
20. Hagiwara Y, Mesch RA, Kawakami T, Okazaki M, Jockusch S, Li YJ, Turro NJ, Wilson CG. J Org Chem. 2013; 78:1730. [PubMed: 23113563]

21. Suyama K, Araki H, Shirai M. J Photopolym Sci Technol. 2006; 19:81.

22. Sun X, Gao JP, Wang ZY. J Am Chem Soc. 2008; 130:8130. [PubMed: 18528981]

23. Zhao L, Vaupel M, Loy DA, Shea K. J Chem Mater. 2008; 20:1870.

24. Xi WX, Peng HY, Aguirre-Soto A, Kloxin CJ, Stansbury JW, Bowman CN. Macromolecules. 2014; 47:6159. [PubMed: 25264379]

25. Xi WX, Krieger M, Kloxin CJ, Bowman CN. Chem Commun. 2013; 49:4504.

26. San Miguel V, Bochet CG, del Campo A. J Am Chem Soc. 2011; 133:5380. [PubMed: 21413802]

27. Ohba T, Suyama K, Shirai M. React Funct Polym. 2006; 66:1189.

28. Chatani S, Gong T, Earle BA, Podgorski M, Bowman CN. ACS Macro Lett. 2014; 3:315.

29. Bao C, Zhu L, Lin Q, Tian H. Adv Mater. 2015; 27:1647. [PubMed: 25655424]

30. Liu ZZ, Lin QN, Sun Y, Liu T, Bao CY, Li FY, Zhu LY. Adv Mater. 2014; 26:3912. [PubMed: 24652710]

31. Kotzur N, Briand B, Beyermann M, Hagen V. J Am Chem Soc. 2009; 131:16927. [PubMed: 19863095]

32. Klan P, Solomek T, Bochet CG, Blanc A, Givens R, Rubina M, Popik V, Kostikov A, Wirz J. Chem Rev. 2013; 113:119. [PubMed: 23256727]

33. Givens RS, Rubina M, Wirz J. Photoch Photobio Sci. 2012; 11:472. 
(a)

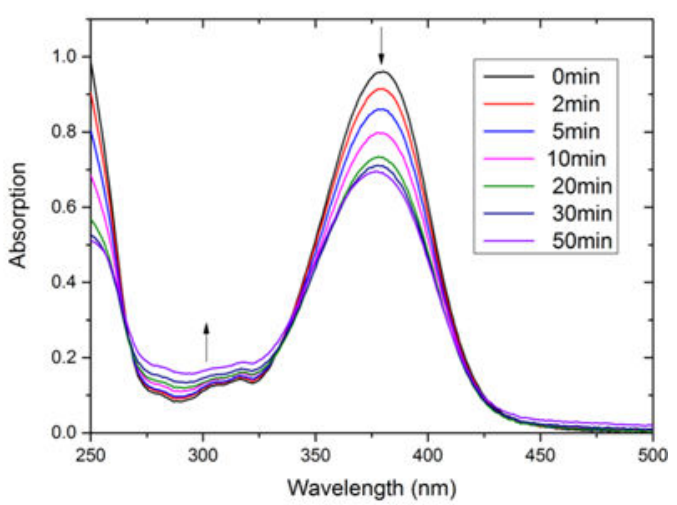

(b)

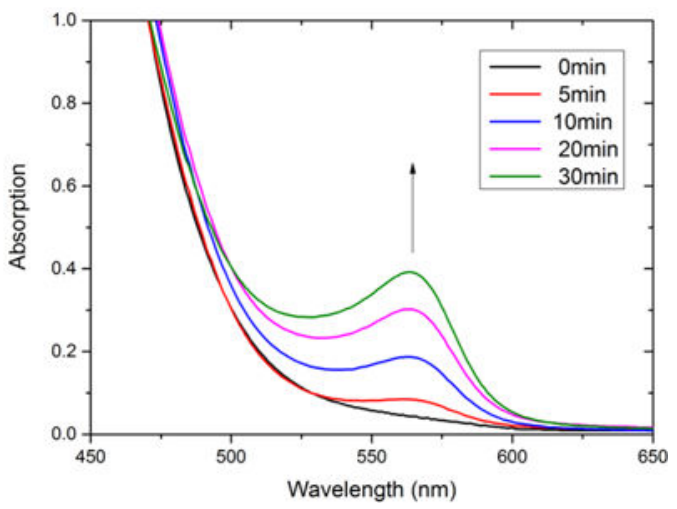

Figure 1.

UV-vis tests on photolysis of coumarin-TMG solutions: (a) the absorption spectrum changes of $0.05 \mathrm{mM}$ coumarin-TMG in methanol with continuous $400-500 \mathrm{~nm}, 50 \mathrm{~mW} / \mathrm{cm}^{2}$ irradiation. (b) Coumarin-TMG methanol solutions were irradiated with 400-500 nm light at $30 \mathrm{~mW} / \mathrm{cm}^{2}$, and $10^{-3} \mathrm{M}$ phenol red solution was added afterward. A new absorption peak appears and increases in intensity with increasing irradiation time. 

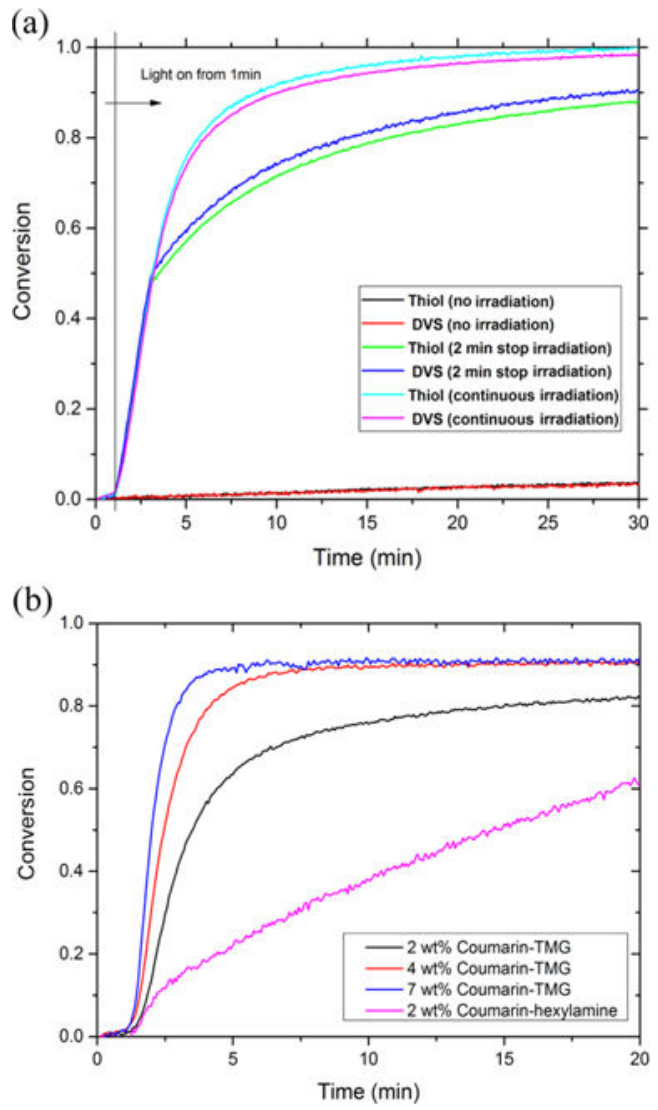

Figure 2.

Conversions as a function of time for stoichiometric DVS/thiol mixtures: (a) thiol and vinyl conversions versus time for butyl thioglycolate and DVS mixture initiated by 2 wt \% coumarin-TMG and initiated with $50 \mathrm{~mW} / \mathrm{cm}^{2}, 400-500 \mathrm{~nm}$ light. The controlled experiment was conducted under continuous irradiation, and the dark cure sample was irradiated for only $2 \mathrm{~min}$ and then monitored after irradiation. (b) Thiol conversion versus reaction time in PETMP/DVS cross-linking systems with different coumarin-TMG loading. All reactions were initiated with $50 \mathrm{~mW} / \mathrm{cm}^{2}, 400-500 \mathrm{~nm}$ irradiation. Also, the coumarinhexylamine-catalyzed polymerization was included for comparison of the catalytic efficiency of each of the photobases. 

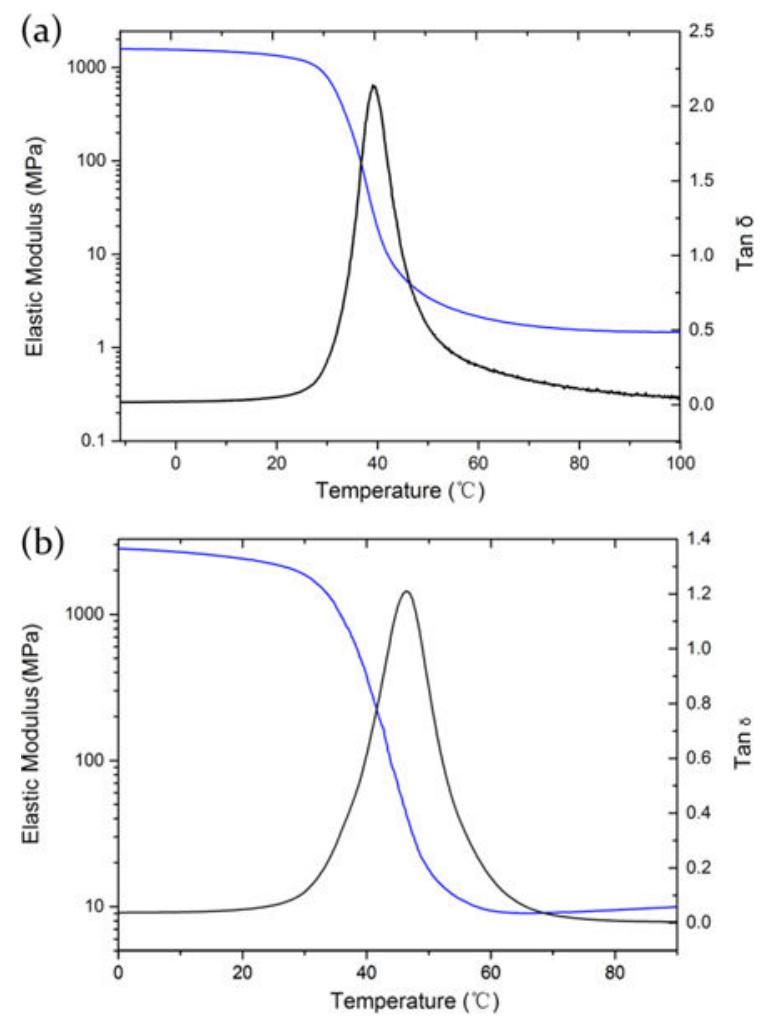

Figure 3.

Tan $\delta$ and elastic modulus profiles for (a) thiol-vinyl stoichiometric cross-linked polymeric film made of PETMP and DVS catalyzed by 2 wt \% coumarin-TMG with $50 \mathrm{~mW} / \mathrm{cm}^{2}, 400-$ $500 \mathrm{~nm}$ irradiation; (b) thiol-epoxy stoichiometric film made of PETMP and bisphenol A diglycidyl ether catalyzed by $2 \mathrm{wt} \%$ coumarin-TMG cured under $50 \mathrm{~mW} / \mathrm{cm}^{2}, 400-500 \mathrm{~nm}$ irradiation conditions. 
(a)
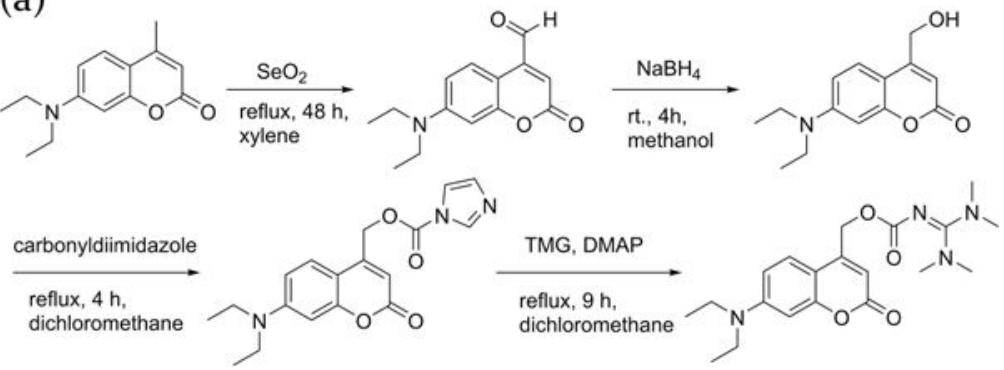

(b)
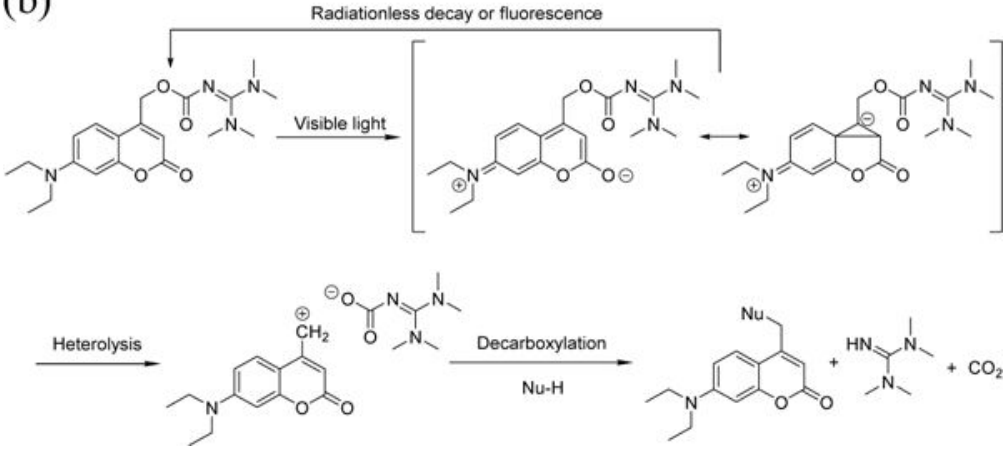

Scheme 1.

(a) Synthetic Route for the Synthesis of Coumarin-TMG and (b) Proposed Coumarin-TMG Photolysis Mechanism upon Visible-Light Irradiation 


\section{Table 1}

Stoichiometric Mixtures of Different Monothiols and Divinyl Sulfone (DVS) Catalyzed with 2 wt \% Coumarin-TMG Irradiated with $50 \mathrm{~mW} / \mathrm{cm}^{2}$ Visible Light of $400-500 \mathrm{~nm}^{\mathrm{a}}$

\begin{tabular}{|c|c|c|c|c|}
\hline entry & thiol & catalyst loading & time (min) & yield $(\%)$ \\
\hline 1 & butyl thioglycolate & $2 \mathrm{wt} \%$ & 20 & 98 \\
\hline 2 & butyl 3-mercaptopropionate & $2 \mathrm{wt} \%$ & 10 & 99 \\
\hline 3 & furan-2-ylmethanethiol & $2 \mathrm{wt} \%$ & 5 & 98 \\
\hline 4 & 1-hexanethiol & $2 \mathrm{wt} \%$ & 5 & 99 \\
\hline 5 & mercaptoethanol & $1 \mathrm{wt} \%$ & 30 & 94 \\
\hline $6^{b}$ & butyl thioglycolate & 0 & 60 & 0 \\
\hline $7^{c}$ & butyl thioglycolate & 2 wt $\%$ & 60 & trace \\
\hline \multicolumn{5}{|c|}{${ }^{a}$ Reaction times to reach the final conversions were measured by FT-IR, and ${ }^{1} \mathrm{H}$ NMR further confirmed the reaction yields. } \\
\hline
\end{tabular}

